\title{
Ocular disease in patients with tuberculosis and HIV presenting with fever in Africa
}

\author{
N A V Beare, J G Kublin, D K Lewis, M J Schijffelen, R P H Peters, G Joaki, \\ J Kumwenda, E E Zijlstra
}

Series editors: W V Good and $S$ Ruit

See end of article for authors' affiliations

Correspondence to: Nicholas Beare, St Paul's Eye Unit, Royal Liverpool University Hospital, Prescot Street, Liverpool, L7 8XP,

UK; nbeare@btinternet.com

Accepted for publication 11 March 2002

\begin{abstract}
Aims: To investigate ocular disease in patients with tuberculosis (TB) and HIV in Africa presenting with fever, and to determine if indirect ophthalmoscopy is useful in the diagnosis of mycobacteraemia. Methods: A prospective study of all adult patients admitted with fever to a large central hospital in

Malawi, Africa. All recruited patients had an ophthalmic examination, HIV tests, chest $x$ ray, sputum examinations, bacterial and mycobacterial blood cultures, and malaria slide to observe the presence of parasites.

Results: 307 patients were recruited; 109 (36\%) had TB, including 53 (17\%) with mycobacteraemia; 255 (83\%) had HIV and 191 (62\%) had AIDS. Of the patients with TB 102 (94\%) had HIV. Choroidal granulomas were found in four patients, all of whom had AIDS; three (2.8\% of those with TB) had disseminated TB with mycobacteraemia, and one had persistent fever but no other evidence of TB. Among the patients with AIDS, $32(17 \%)$ had microangiopathy manifest by cotton wool spots; one $(0.5 \%)$ had signs of active cytomegalovirus (CMV) retinitis. The presence of microangiopathy was not related to TB. Conclusions: In Malawian patients with TB presenting acutely with fever, choroidal granulomas were found in $2.8 \%$, and were concurrent with mycobacteraemia and AIDS. Ophthalmoscopy was not a useful aid in the diagnosis of mycobacteraemia. Cytomegalovirus (CMV) retinitis is rarely seen in African AIDS patients. This may be the result of mortality early in the disease course, or differences in race, HIV subtype, or comorbidity.
\end{abstract}

en $\mathrm{n}$ the past 15 years sub-Saharan Africa has seen an enormous increase in tuberculosis (TB) cases, largely as a result of associated of HIV infection. HIV increases the development of active TB to around $30 \%$ of subjects infected with tubercle bacilli. ${ }^{1}$ Furthermore, TB is the most frequent cause for admission of HIV patients in Africa; but data regarding the ocular consequences of co-infection with HIV and TB are limited. ${ }^{2}$ Two thirds of AIDS patients in Malawi and Burundi examined for ocular disease had TB, but lesions clearly associated with TB were not seen. ${ }^{34}$

Bloodstream infection with Mycobacterium tuberculosis (mycobacteraemia) has recently been recognised as a common cause of fever in Africans admitted to hospital. ${ }^{5-8}$ However, culture of $M$ tuberculosis from blood requires specialist techniques and prolonged incubation. Any cheap, practical tools which aid in the diagnosis of mycobacteraemia, such as ophthalmoscopy for choroidal granulomas, would be useful in this setting. Choroidal granulomas are presumed to spread haematologically, but the ocular manifestations of TB have not previously been examined in relation to mycobacteraemia.

Ocular complications of AIDS are common, occurring in $70-80 \%$ of patients in Western countries before the advent of highly active antiretroviral therapy (HAART). ${ }^{9}$ However, the greatest burden of HIV is in sub-Saharan Africa where at least 30 million people are infected, with poor health care and no access to antiviral drugs. ${ }^{10}$

The available data suggest that the spectrum of ocular disease caused by AIDS is different in Africa, with CMV retinitis being less common, and herpes zoster ophthalmicus and conjunctival squamous cell tumours being more prevalent. ${ }^{2} \mathrm{CMV}$ retinitis has been found to occur in $0-8.5 \%$ of AIDS patients in Africa. ${ }^{11}$ The rates of HIV related microangiopathy, manifest as cotton wool spots (CWS) and small retinal haemorrhages, in AIDS patients are reported as $8 \%$ and $25 \%$ in west Africa, ${ }^{12}{ }^{13}$ $10 \%$ in Burundi, ${ }^{4}$ and $13 \%$ in Malawi. ${ }^{3}$ The prevalent HIV type in Malawi is almost exclusively HIV-1, in common with other countries in east and southern Africa.

This study was undertaken as part of an investigation of mycobacteraemia in medical patients, with the primary intention of determining whether ocular fundus examination was useful in the diagnosis of mycobacteraemia. We prospectively studied all adults admitted to medical wards with fever in order to also determine the prevalence and type of ocular manifestations of TB, HIV, and AIDS seen in this population.

\section{PATIENTS AND METHODS}

The study recruited adults with a fever or history of fever admitted to the medical wards of Queen Elizabeth Central Hospital, Blantyre, Malawi. This is a large central hospital serving the city of Blantyre and surrounding districts. Patients were eligible if over 13 years old, with either an axillary temperature $\geqslant 37.4^{\circ} \mathrm{C}$ or a history of high fever in the preceding 4 days. They were recruited in 24 hour periods planned over 11 weeks so as to avoid overwhelming clinical staff and laboratory facilities. Recruitment was on a total of 21 days and the actual days of each week were rotated.

The study was explained to all eligible people in their own language, and consent obtained if they agreed. Those recruited had a clinical examination and laboratory investigations including three sputum samples examined for acid fast bacilli, chest $x$ ray, bacterial and mycobacterial blood cultures, two rapid enzyme linked immunosorbent assays (ELISA) HIV tests (Uni-Gold, Trinity Biotech, Bray, Ireland, and Abbot Determine, Dainabot Company, Tokyo, Japan), full blood count, and blood film for malaria parasites. The HIV tests did not differentiate HIV-1 and HIV-2 antibodies, and two were used in order to minimise false positive or negative results. Treatment and further investigations were guided by normal clinical practice. Laboratory investigations were undertaken by the Department of Medicine, Malawi College of Medicine and the Wellcome Trust Research Laboratories, Blantyre. 


\begin{tabular}{ll}
$\begin{array}{l}\text { Table } 1 \text { External eye and anterior } \\
\text { segment findings }\end{array}$ \\
\hline $\begin{array}{ll}\text { External and anterior } \\
\text { segment findings }\end{array}$ & $\begin{array}{l}\text { Number of } \\
\text { patients }\end{array}$ \\
\hline Corneal scar & 6 \\
Conjunctival tumour & $3^{*}$ \\
Posterior synechiae & $3^{*}$ \\
\hline *All patients had AIDS. & \\
\hline
\end{tabular}

Patients had an ocular examination during the first 24 hours of their admission by an ophthalmologist (NB) who was not aware of their diagnosis. This consisted of a bedside examination of eye movements, and torch examination of the external eye, anterior segment, and pupillary reactions. Posterior synechiae were noted but microscopic signs of previous or active uveitis would not have been detected. The fundus was examined by direct and indirect ophthalmoscopy after pupil dilatation with tropicamide $1 \%$ and phenylephrine $2.5 \%$.

The study was approved by the Malawi College of Medicine research committee and the tenets of the Declaration of Helsinki were observed.

Differences between proportions were examined for significance by a $\chi^{2}$ test.

\section{RESULTS}

There were 634 people admitted on study days, of whom 347 were eligible for the study, and 334 were recruited. Ocular examinations were not done on 27 patients owing to death, discharge, or unavailability of patients; 307 (92\%) had an ocular examination, and the remaining analysis is on these patients.

The mean age was 32 years (range 14-64), and 55\% were women. The number of patients with TB was 109 (36\%), including 53 (17\%) with positive mycobacterial blood cultures. A total of 255 (83\%) patients were HIV positive and 191 (62\%) had AIDS. AIDS was defined by the expanded World Health Organization (WHO) case definition for AIDS surveillance, ${ }^{14}$ or stage 3 or 4 of HIV disease according to the WHO staging system. ${ }^{15}$ By these definitions TB is an AIDS defining illness in the presence of HIV. Of patients with TB, 102 (94\%) had HIV and therefore AIDS. Patients with TB were 53\% of all AIDS cases.

\section{External eye and anterior segment findings}

The results of external eye and anterior segment examination on 307 patients are given in Table 1. Two of the conjunctival tumours were excised and examined histologically. They were reported as carcinoma in situ, and "squamous cell hyperplasia, with mild to moderate dysplasia."

Posterior synechiae were seen in three patients with AIDS. One had mycobacteraemia, but no others had TB.

There were no patients with skin stigmata of previous herpes zoster ophthalmicus.

\section{Neuro-ophthalmological findings}

Optic disc findings are given in Table 2. Eleven (4\%) patients were judged to have papilloedema; of these eight had AIDS ( $4 \%$ of those with AIDS), and two $(2 \%)$ had TB, including one with TB meningitis. Two patients with pulmonary TB had unilateral disc swelling, both had AIDS, and neither had evidence of disseminated TB.

Two $(0.7 \%)$ patients with AIDS were diagnosed with cryptococcal meningitis by India ink stain of cerebrospinal fluid, one had a normal ocular examination and one had papilloedema and a sixth cranial nerve palsy.

\section{Chorioretinal findings}

Four people, all with AIDS, had clinically suspected choroidal TB granulomas (Table 3 ). Three had TB including mycobacteraemia. The fourth person was given a therapeutic trial of TB treatment, because of persistent unexplained fever not responding to broad spectrum antibiotics. He had no other findings specific to $\mathrm{TB}$, or other causes of choroidal granuloma.

Chorioretinal findings are given in Table 4. Cotton wool spots (CWS), with or without small retinal haemorrhages, in the presence of HIV were regarded as microangiopathy. Thirty five ( $14 \%$ ) of all patients with HIV, and 32 (17\%) of those with AIDS, had microangiopathy. Three (5\%) patients with HIV, but without AIDS defining features, had microangiopathy (Table 5).

There was no association between the presence of microangiopathy and TB $\left(\chi^{2} \mathrm{p}=0.72\right)$. The proportion of AIDS patients who had microangiopathy was larger in the group with mycobacteraemia than in those without mycobacteraemia, but this was not statistically significant $\left(\chi^{2} \mathrm{p}=0.18\right)$.

Two patients had CWS but did not have HIV, including one with TB abdominal adenitis and mycobacteraemia, and one with heart failure secondary to rheumatic heart disease (single CWS).

\begin{tabular}{|c|c|c|c|c|c|}
\hline \multirow[b]{2}{*}{ Disc finding } & \multirow{2}{*}{$\begin{array}{l}\begin{array}{l}\text { Number of } \\
\text { patients }\end{array} \\
\text { No (\%) }\end{array}$} & \multirow[b]{2}{*}{ Diagnosis } & \multicolumn{3}{|c|}{ Number of patients } \\
\hline & & & All & HIV+ & AIDS \\
\hline $\begin{array}{l}\text { Normal } \\
\text { Bilateral disc swelling/papilloedema }\end{array}$ & $282(92)$ & & & & \\
\hline \multirow{6}{*}{ Bilateral disc swelling/papilloedema } & $11(3.6)$ & Cryptococcal meningitis and TB & 1 & 1 & 1 \\
\hline & & TB meningitis & 1 & 1 & 1 \\
\hline & & Bacterial meningitis & 2 & 1 & 1 \\
\hline & & Sepsis or pneumonia & 4 & 3 & 2 \\
\hline & & Malaria & 1 & 1 & 1 \\
\hline & & Pyrexia of unknown origin & 2 & 2 & 2 \\
\hline \multirow{3}{*}{ Hyperaemic discs } & $7(2.3)$ & & & & \\
\hline & & Malaria & 4 & 3 & 0 \\
\hline & & Pneumonia/sepsis & 3 & 3 & 2 \\
\hline \multirow[t]{2}{*}{ Unilateral disc swelling } & $2(0.7)$ & & & & \\
\hline & & Pulmonary TB & 2 & 2 & 2 \\
\hline \multirow[t]{3}{*}{ Disc haemorrhages } & $2(0.7)$ & & & & \\
\hline & & Sepsis & 1 & 1 & 1 \\
\hline & & TB adenitis and mycobacteraemia & 1 & 0 & 0 \\
\hline \multirow[t]{3}{*}{ Other } & 3 & & & & \\
\hline & & Disc drusen & 2 & & \\
\hline & & Myelinated fibres & 1 & & \\
\hline
\end{tabular}


Table 3 Patients with choroidal granulomas

\begin{tabular}{lllllll}
\hline Patient & Sex & $\begin{array}{l}\text { M tuberculosis in } \\
\text { sputum }\end{array}$ & Other findings & $\begin{array}{l}\text { Mycobacterial } \\
\text { blood cultures }\end{array}$ & $\begin{array}{l}\text { AIDS } \\
\text { status }\end{array}$ & $\begin{array}{l}\text { Number of } \\
\text { granulomas }\end{array}$ \\
\hline 1 & F & Positive & Pericardial effusion & Positive & AIDS & 1 \\
2 & $M$ & Positive & TB changes on CXR* & Positive & AIDS & 4 \\
3 & F & Negative & Miliary TB on CXR & Positive & AIDS & 5 \\
4 & $M$ & Negative & Persistent fever & Negative & AIDS & 2 \\
\hline CXR = chest $x$ ray. & & & & & \\
*Infiltration in all 6 zones.
\end{tabular}

Table 4 Chorioretinal findings in all patients, patients with AIDS, and patients with $\mathrm{TB}$

\begin{tabular}{llll}
\hline & \multicolumn{4}{l}{ Number of patients (\%) } \\
\cline { 2 - 4 } Retinal finding & All & AIDS & TB \\
\hline Normal & $243(79)$ & $140(73)$ & $84(77)$ \\
CWS alone & $29(9)$ & $23(13)$ & $17(16)$ \\
CWS and retinal haemorrhage & $9(3)$ & $8(4)$ & $2(2)$ \\
Choroidal granuloma & $4^{*}(1)$ & $4^{*}(2)$ & $3(3)$ \\
Active retinitis & $1(0.3)$ & $1(0.5)$ & $0(0)$ \\
$1-4$ retinal haemorrhages & $5(1)$ & $2(0)$ & $0(0)$ \\
$>25$ retinal haemorrhages & $2(0.7)$ & $2(1)$ & $0(0)$ \\
Scars and incidental findings & $16(5)$ & $11(6)$ & $3(3)$ \\
Total & 307 & 191 & 109 \\
\hline CWS = cotton wool spot.. & & & \\
*One also had CWS. & & & \\
\hline
\end{tabular}

Table 5 Proportion of patients with microangiopathy in different groups

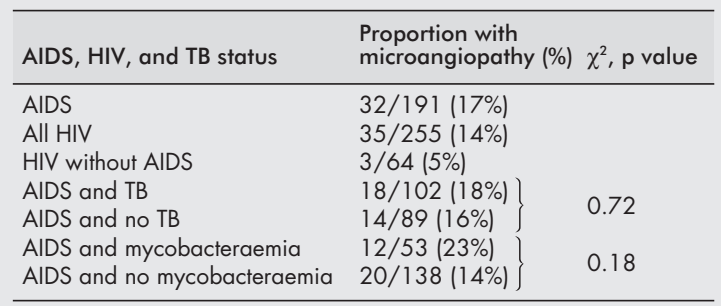

One $(0.5 \%)$ patient with AIDS had widespread bilateral retinitis with opacified retina, confluent haemorrhages, CWS, and vascular sheathing consistent with a CMV retinitis.

Two patients had a large number of discrete retinal haemorrhages, numbering approximately 30 in each eye, over $50 \%$ of which were white centred blot haemorrhages. Both had AIDS and were severely anaemic with haemoglobin values of 3.0 and $3.8 \mathrm{~g} / \mathrm{dl}$ (median haemoglobin of all patients was $9.6 \mathrm{~g} / \mathrm{dl}$, interquartile range $7.4-11.6 \mathrm{~g} / \mathrm{dl}$ ). In each case no malaria parasites were found on a single slide examination which makes clinical malaria in immune adults extremely unlikely.

\section{Incidental retinal findings}

Sixteen $(5 \%)$ patients had incidental retinal findings including macular and peripheral chorioretinal scars, epiretinal membrane, and macular hole. One patient had peripheral retinal bone spicule pigmentation with attenuated vessels characteristic of retinitis pigmentosa. Two patients had retinal signs associated with severe malaria. ${ }^{16}$

\section{DISCUSSION}

This prospective study of ocular disease in Malawian patients presenting with fever includes comprehensive data on TB, mycobacteraemia, and HIV. We found presumed choroidal granulomas in $2.8 \%$ of febrile Malawians with proved TB. All three TB patients with choroidal granulomas had disseminated TB with $M$ tuberculosis bloodstream infection (mycobacteraemia). In all there were 53 patients (49\% of TB cases) with mycobacteraemia recognised using specialised blood culture techniques with prolonged incubation. This is the first time that choroidal granulomas have been linked to mycobacteraemia, and the finding is consistent with the presumed haematological spread of bacilli found in choroidal tubercles. ${ }^{17} 18$

Modern data on the prevalence of choroidal granulomas in TB are extremely limited, particularly since the development of AIDS or even the introduction of the indirect ophthalmoscope. Choroidal tubercles were reported in $28 \%$ of 737 patients with miliary $\mathrm{TB}$ reviewed in the literature by Illingworth and Wright in 1948, although they found $60 \%$ in 42 miliary TB patients. ${ }^{19}$ Series of patients in TB sanitariums report choroidal granulomas only rarely. ${ }^{20}{ }^{21}$

Our figure suggests the prevalence of choroidal granulomas in acute medical admissions with TB in Africa is somewhere in between. This figure reflects the relatively high prevalence of disseminated TB associated with HIV and immunosuppression. In this study patients with TB mainly had pulmonary TB, but there were eight patients with chest $x$ ray changes of miliary TB and a high proportion (49\%) with disseminated TB in the form of bloodstream infection. It should be noted that one patient with suspected choroidal granuloma had no evidence for TB apart from persistent fever.

We found microangiopathy in $17 \%$ of patients with AIDS, which is similar to, although slightly higher than, other studies in central and eastern Africa. ${ }^{34}$ Microangiopathy was also present in $5 \%$ of adult medical admissions with HIV, but without AIDS defining illnesses. Morgan et al found no microangiopathy in 105 people with HIV living in the community in Uganda, ${ }^{22}$ but patients in our study are likely to be further in the disease progression than the HIV population at large.

The presence of microangiopathy was not related to TB. AIDS patients with mycobacteraemia tended to have more 
retinal microangiopathy, but this was not statistically significant. A higher prevalence of microangiopathy in patients with mycobacteraemia may reflect their overall worse state of health.

Although HIV microangiopathy is the commonest cause of CWS, it is important to recognise that not all patients in African medical wards with CWS and small blot retinal haemorrhages have HIV.

The number of patients with posterior synechiae is too small to draw any firm conclusions. However, only one patient with this sign of previous anterior uveitis had TB, and this was detected by mycobacteraemia alone. All patients with posterior synechiae had AIDS, which may account for the relatively high prevalence of anterior uveitis in general hospital admissions. HIV infection has been associated with uveitis in 170 patients in Zimbabwe. ${ }^{23}$

We found CMV retinitis to be very uncommon, in keeping with other surveys of patients with AIDS in Africa. CMV antibodies are widespread in this population, appearing in $89 \%$ of antenatal women (E Molyneux, personal communication). The reason for the rarity of CMV retinitis in Africa is generally considered to be due to mortality of people with AIDS early in the disease progression before, or shortly after, the onset of profound immunosuppression. ${ }^{3411}$ The prevalence of CMV retinitis in pre-HAART American AIDS patients was 20\%, 2 years after their CD4 count fell below $100 \times 10^{6} / 1 .{ }^{24} \mathrm{CD} 4$ counts have been found to be below $100 \times 10^{6} / 1$ in as many as $25 \%$ of hospitalised AIDS patients in Africa, ${ }^{4}$ but accelerated disease progression may mean that their life expectancy is short, and hence CMV retinitis is rarely seen.

A second possibility is that differences in race, HIV subtype, and comorbidity may influence the occurrence of CMV retinitis in Africa. This hypothesis is strengthened by a study of 313 Africans with AIDS living in London. ${ }^{25}$ Although the prevalence of CMV disease at presentation in Africans was equal to controls (4\%), the Africans' mean CD4 count was lower than controls $\left(25 \times 10^{6} / 1\right.$ versus $\left.45 \times 10^{6} / 1\right)$, and the number subsequently developing CMV disease was significantly lower, despite treatment at the same centres. It remains a possibility that other factors apart from life expectancy influence the development of CMV retinitis. However, unless life expectancy dramatically improves for patients with AIDS in Africa it would be difficult to demonstrate the effect of other factors.

This study has demonstrated that choroidal granulomas occur concurrently with bloodstream infection with $M$ tuberculosis, and that the rate of choroidal granulomas is $2.8 \%$ in African TB patients presenting acutely with fever. Examining fundi for choroidal granulomas is not a useful aid in the diagnosis of mycobacteraemia, but their presence can confirm a diagnosis of disseminated TB.

\section{ACKNOWLEDGEMENTS}

This study was funded by the Malawi Health Support Fund of the Royal Netherlands Embassy in Lusaka (grant no MW004401/02), and by the National TB Programme of Malawi. NB was funded by the Foundation for the Prevention of Blindness, Liverpool, UK.

We thank the patients, relatives, and guardians for participating in this study. We also thank our dedicated clinical staff, particularly Ledson Mkwaira, Freda Nsamala, Mercy Mtegha, Gabriel Matheyu, and Jonathan Waluza; and also the staff of wards 4A and 3B of Queen Elizabeth Central Hospital, Blantyre. We gratefully acknowledge the work of the laboratory staff of the Wellcome Trust Research Laboratories, in particular Mandy Walsh; and the Department of Medicine,
Malawi College of Medicine, Blantyre. We would also like to thank the staff of the Eye Department, QECH, for loan of equipment and facilities. Simon Harding kindly read and provided comments on the manuscript.

\section{Authors' affiliations}

N A V Beare, St Paul's Eye Unit, Royal Liverpool University Hospital, Liverpool, UK and Wellcome Trust Research Laboratories, Blantyre, Malawi

J G Kublin, Department of Community Health, College of Medicine, University of Malawi, Blantyre, Malawi

D K Lewis, M J Schijffelen, R P H Peters, J Kumwenda, E E Zijlstra,

Department of Medicine, College of Medicine, University of Malawi

G Joaki, Department of Microbiology, College of Medicine, University of Malawi

\section{REFERENCES}

1 Styblo K. The impact of HIV infection on the global epidemiology of tuberculosis. Bull Int Union Tuberc Lung Dis 1991;66:27-32

2 Lewallen S, Courtright P. HIV and AIDS and the eye in developing countries. Arch Ophthalmol 1997;115:1291-5.

3 Lewallen S, Kumwenda J, Maher D, et al. Retinal findings in Malawian patients with AIDS. Br J Ophthalmol 1994;78:757-9.

4 Cochereau I, Mlika-Cabanne N, Godinaud P, et al. AIDS related eye disease in Burundi, Africa. Br J Ophthalmol 1999;83:339-42

5 Archibald LK, den Dulk MO, Pallangyo KJ, et al. Fatal Mycobacterium tuberculosis bloodstream infections in febrile hospitalized adults in Dar es Salaam, Tanzania. Clin Infect Dis 1998:26:290-6.

6 Ssali FN, Kamya MR, Wabwire Mangen F, et al. A prospective study of community-acquired bloodstream infections among febrile adults admitted to Mulago Hospital in Kampala, Uganda. J Acquir Immune Defic Syndr Hum Retrovirol 1998;19:484-9.

7 McDonald LC, Archibald LK, Rheanpumikankit S, et al. Unrecognised Mycobacterium tuberculosis bacteraemia among hospital inpatients in less developed countries. Lancet 1999:354:1159-63.

8 Archibald LK, McDonald LC, Nwanyanwu O, et al. A hospital-based prevalence survey of bloodstream infections in febrile patients in Malawi: implications for diagnosis and therapy. J Infect Dis 2000;181:1414-20.

9 Cunningham ET, Margolis TP. Ocular manifestations of HIV infection. N Engl J Med 1998;339:236-44.

10 UNAIDS/WHO. AIDS epidemic update: December 2000. Geneva. World Health Organization, 2000

11 Kestelyn P. The epidemiology of CMV retinitis in Africa. Ocul Immunol Inflamm 1999;7:173-7.

12 Jaffar S, Ariyoshi K, Frith $\mathrm{P}$, et al. Retinal manifestations of HIV-1 and HIV-2 infections among hospital patients in the Gambia, West Africa. Trop Med Int Health 1999;4:487-92.

13 Balo KP, Fany A, Amoussou YP, et al. Les complications retiniennes chez des patients attients de SIDA au CHU de Lome (Togo). J Fr Ophtalmol 1998;21:435-9.

14 World Health Organization. WHO case definitions for AIDS surveillance in adults and adolescents. Weekly Epidem Rec 1994:69:273-5.

15 World Health Organization. Acquired immunodeficiency syndrome (AIDS). Interim proposal for a WHO staging system for HIV infection and disease. Weekly Epidem Rec 1990;65:22 1-4

16 Lewallen S, Harding SP, Ajewole J, et al. A review of the spectrum of clinical fundus findings in P falciparum malaria in African children with a proposed classification and grading system. Trans Roy Soc Trop Med Hyg 1999;93:619-22.

17 Yanoff M, Fine BS. In: Ocular pathology. 4th ed. London: Mosby-Wolfe, 1996:76-7.

18 Helm CJ, Holland GN. Ocular tuberculosis. Surv Ophthalmol 1993;38:229-56

19 Illingworth RS, Wright T. Tubercles of the choroid. BM 1948;2:365-8

20 Duke-Elder S, Perkins ES. Bacterial uveitis: tuberculosis. In: Duke-Elder S, ed. System of ophthalmology. Vol IX. 1st ed. London: Henry Kimpton, 1966:249-57.

21 Dinning WJ, Marston S. Cutaneous and ocular tuberculosis: a review. J Roy Soc Med 1985;78:576-81.

22 Morgan D, Jones C, Whitworth J, et al. Ocular findings in HIV-1 positive and HIV-1 negative participants in a rural population-based cohort in Uganda. Int Ophthalmol 1999;22:183-92

23 Ronday MJH, Welbeck RO, Chana HS, et al. Analysis of infectious causes of uveitis in Zimbabwe using aqueous humor analysis. In: Ronday MH. Uveitis in Africa. Amsterdam: Netherlands Ophthalmic Research Institute, 1996.

24 Hoover DR, Yun Peng MS, Saah A, et al. Occurrence of cytomegalovirus retinitis after human immunodeficiency virus immunosuppression. Arch Ophthalmol 1996;114:821-7.

25 Del Amo J, Petruckevitch A, Phillips AN, et al. Spectrum of disease in Africans with AIDS in London. AIDS 1996;10:1563-9 